\title{
Triple-Negative Breast Cancer: What Crucial Information can Imaging Add to the Diagnosis, Treatment and Prognosis?
}

\author{
William M McGarry ${ }^{1,2}$ and Sonya Bhole ${ }^{1^{*}}$ \\ ${ }^{1}$ Department of Radiology, Lynn Sage Comprehensive Breast Center, Prentice Women's Hospital, Feinberg \\ School of Medicine, Northwestern University, Chicago, USA \\ ${ }^{2}$ Radiology Associates of Wyoming Valley, Wilkes-Barre, USA
}

*Corresponding author: Sonya Bhole, MD, Lynn Sage Breast center, Northwestern Memorial Hospital, 250 E superior street Chicago, IL 60611, USA

\begin{abstract}
Breast cancer is a heterogeneous disease, with many distinct subtypes having extremely different presentations, biological aggressiveness, and response to therapy. Triplenegative breast cancer is a subtype with significant clinical implications because of its poor prognosis and aggressive behavior. It has distinct imaging features, often presenting as a mass with typically benign features on mammography and ultrasound and more suspicious findings on magnetic resonance imaging. Triple-negative breast cancer also has unique treatment limitations, with these cancers lacking molecular therapeutic targets expressed by other breast cancer subtypes. Fortunately, Triple-negative breast cancer is very responsive to neoadjuvant chemotherapy, which is the mainstay of treatment for these patients. Patients with triple-negative breast cancer are more likely to achieve pathologic complete remission, which is associated with improved survival. Despite this, patients with triple-negative breast cancer have an overall poorer prognosis compared to other subtypes, especially in the first few years after diagnosis. As this subtype accounts for up to one-fifth of all breast cancer cases, a thorough understanding of the unique imaging features of triple-negative breast cancer, as well as the role of imaging in predicting response to therapy and long term patient prognosis, will allow all involved clinicians to more effectively care for these patients.
\end{abstract}

\section{Keywords}

Breast cancer, Triple negative, Mammography, Neoadjuvant chemotherapy, Pathologic complete remission

\section{Introduction}

Breast cancer is the most common malignancy among women in developed countries and is a leading cause of cancer death [1]. It is a heterogeneous disease, with many biologically distinct subtypes having widely different clinical presentations, biological aggressiveness, and responses to therapy $[1,2]$. Triple negative breast cancer (TNBC) is one subtype that is of particular interest due to its poor prognosis, aggressive biology, and associations with TP53 gene mutations and suppressed BRCA1 function [3]. TNBC is defined as a breast cancer that does not express receptors for estrogen (ER), progesterone (PR), or human epidermal growth factor 2 (HER2) [3-5]. TNBC comprises $11-20 \%$ of all breast cancers $[3,6,7]$ but is responsible for $23-28 \%$ of locally advanced disease $[3,8,9]$. It also has distinctive imaging features [3,10-13], often lacking the suspicious mammographic findings associated with other forms of breast cancer. Since TNBC lacks a molecular target, options for systemic therapy are limited as compared to tumors which express ER, PR, and HER2 [3]. Because of its aggressive behavior, unique imaging features, and treatment limitations, it is important that all clinicians caring for breast cancer patients have a broad understanding of TNBC. This review will provide an overview of this topic with a focus on the imaging features, treatment implications, and prognostic factors relevant to TNBC.

\section{Imaging Features}

\section{Mammography}

TNBC usually lacks the spiculated margins, irregular shape, and suspicious calcifications associated with

Citation: McGarry WM, Bhole S (2019) Triple-Negative Breast Cancer: What Crucial Information can Imaging Add to the Diagnosis, Treatment and Prognosis?. Int J Womens Health Wellness 5:087. doi. org/10.23937/2474-1353/1510087

Accepted: January 01, 2019: Published: January 03, 2019

Copyright: (C) 2019 McGarry WM, et al. This is an open-access article distributed under the terms of the Creative Commons Attribution License, which permits unrestricted use, distribution, and reproduction in any medium, provided the original author and source are credited. 


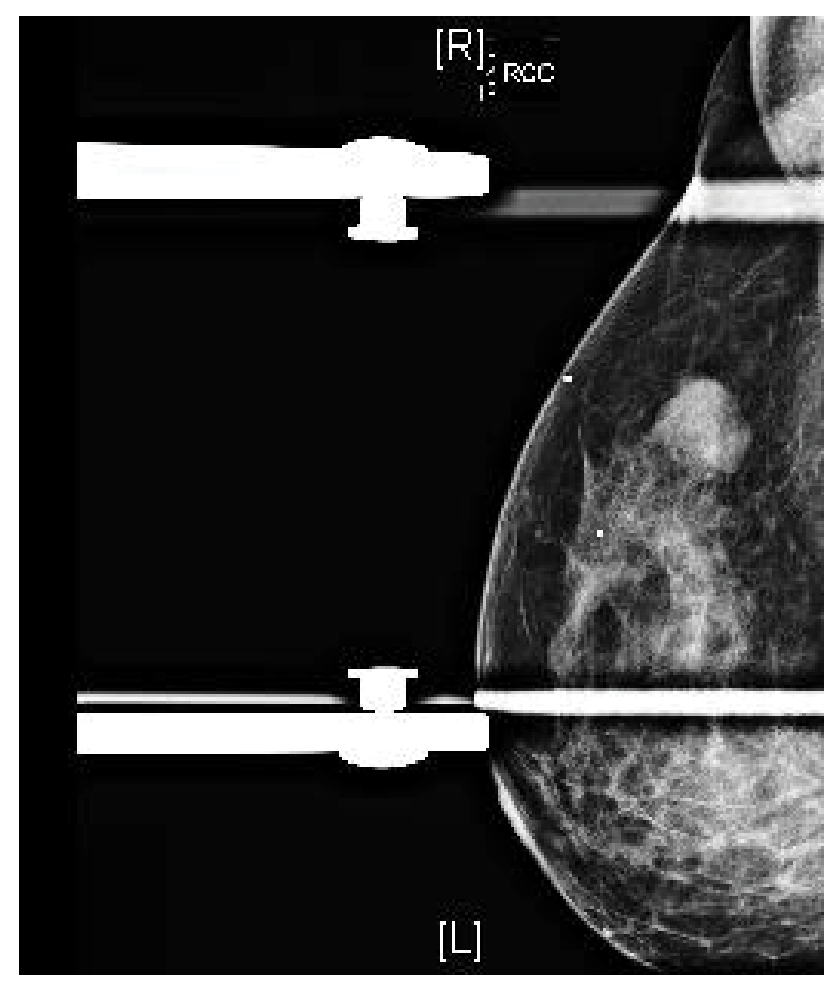

Figure 1: Mammography findings in triple-negative breast cancer (TNBC). Right mediolateral oblique view with spot compression demonstrates an oval mass with circumscribed margins, representing a biopsy-proven TNBC with necrosis.

other forms of breast cancer. As such, TNBC can be mammographically occult (in up to $18 \%$ of cases), despite often being larger than other breast cancers at the time of diagnosis [3]. The most common presentation of TNBC on mammography is a mass $[3,10,12,13$ ] (Figure 1). Circumscribed margins are described in approximately one-fourth of cases $[3,10,12,13]$, and there are typically no associated calcifications $[3,13]$. Less common presentations of TNBC include a focal asymmetry, seen in $10-20 \%$ of cases, or a mass with associated calcifications, seen in approximately $15 \%$ of cases $[11,13]$. Isolated calcifications are a much less frequent presentation $[10,11]$. Dogan, et al. state that mammography alone may have limited value in screening patients at risk for TNBC [3]. These authors indicate that the low incidence of associated calcifications or ductal carcinoma in situ suggests rapid tumor growth that proceeds directly to invasive cancer, without an in situ stage [3].

\section{Ultrasound}

Ultrasound has a very high sensitivity for the detection of TNBC [3]. The most frequent presentation of TNBC on ultrasound is as a discrete mass which, as on mammography, largely lacks suspicious sonographic features (Figure 2). The predominant features of TNBC include well-circumscribed margins, reported in approximately $25 \%$ of cases [3,11-13], and posterior acoustic enhancement, present in $25-40 \%$ of cases $[3,12,13]$. The presence of posterior acoustic enhancement in TNBC suggests tumor necrosis, rather than indicating benignity as in other breast lesions

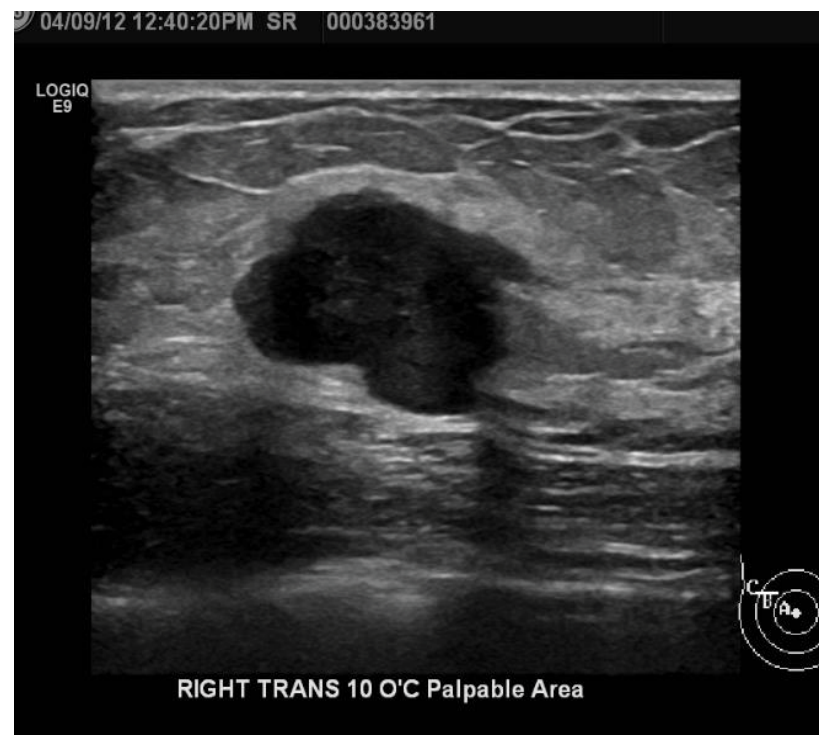

Figure 2: Ultrasound findings in triple-negative breast cancer (TNBC). Right breast ultrasound demonstrates an oval, hypoechoic mass with circumscribed margins and associated posterior acoustic enhancement, representing a biopsy-proven TNBC.

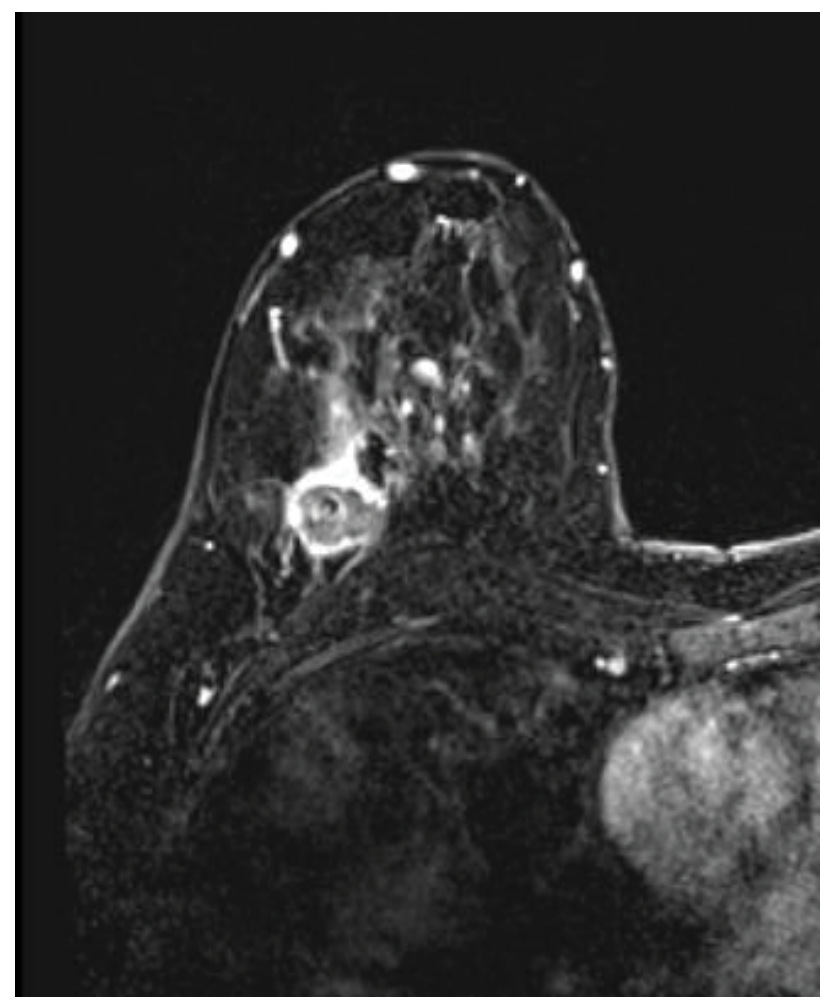

Figure 3: Magnetic resonance findings in triple-negative breast cancer (TNBC). Post-contrast axial and coronal T1 fat-suppressed images of the bilateral breasts demonstrate a round mass with circumscribed margins and rim enhancement in the right breast at posterior depth, representing a biopsy-proven TNBC.

$[3,14,15]$.

\section{Magnetic resonance}

Magnetic resonance imaging (MRI) is extremely sensitive for the detection of TNBC, with the morphologic characteristics of TNBC on MRI more suspicious than those seen on mammography or ultrasound (Figure 3) 
[16]. In a study examining forty-four patients with TNBC, Dogan, et al. found that MRI was $100 \%$ sensitive for the detection of these tumors as compared to sensitivities of $91 \%$ and $93 \%$ for mammography and ultrasound, respectively [13]. The predominant presentation of TNBC in that study was an enhancing mass, seen in 34 patients. The most commonly reported mass shape was round or oval, described in $35 \%$ of patients, with dominant mass margins reported as irregular or spiculated in $47 \%$ and $41 \%$ of cases respectively. The most frequent contrast enhancement pattern was rim enhancement, present in $76 \%$ of cases. Enhancing internal septations were seen in 8 patients. The other 10 patients in this study showed non-mass enhancement. A study by Uematsu, et al. [17] reviewed 59 patients and demonstrated that findings of a mass lesion, rim enhancement pattern, smooth mass margins, and persistent enhancement kinetics were associated with TNBC [17]. Of these findings, Teifke, et al. [18] suggest that rim enhancement was the most accurate MR finding for determining ER status. Although there is a strong association between unifocal lesions and TNBC [17], multifocality has been reported in the literature in $21 \%$ of cases [19]. TNBC tend to be larger in size on MRI than other subtypes, with an average tumor size of $4.1+-2.7 \mathrm{~cm}$ [19]. Prominent skin enhancement was also a frequent finding in this study, suggesting invasion of the dermal lymphatics [19]. High intratumoral T2 signal intensity, which is also associated with TNBC $[13,17,19-21]$, has been shown to be strongly associated with intratumoral necrosis [20]. Osman, et al. demonstrated a $90 \%$ correlation of high T2 signal on MRI with internal necrosis on pathologic examination [20]. This is clinically significant, as internal necrosis is related to poor clinical outcomes and aggressive tumor biology [22]. The presence of necrosis has been associated with reduced relapse free-survival as well as increased mortality in both node-positive and node-negative patients $[22,23]$.

\section{Treatment}

In TNBC, there is no dominant molecular factor supporting tumor growth [3]. Patients with TNBC thus lack the targeted therapy options available to those with hormone and HER2-receptor positive tumors [3,24]. As such, chemotherapy is the mainstay for the systemic treatment of TNBC [24]. Neoadjuvant chemotherapy is currently the standard treatment for patients with larger tumors $(>2.0 \mathrm{~cm}$ ) and locally advanced disease [25]. Neoadjuvant treatment offers several distinct surgical and oncological advantages. From a surgical perspective, neoadjuvant treatment can reduce tumor size, allowing patients to become candidates for surgical resection when they were previously ineligible [25]. It also can convert some patients from mastectomy to breast conservation therapy [25] and improve cosmetic results in patients who were already lumpectomy candidates [26]. A systematic review by Mieog, et al. of patients with operable breast cancer demonstrated that patients undergoing neoadjuvant treatment had a lower mastectomy rate than those receiving surgery before adjuvant therapy, with a relative risk of 0.71 [27]. Neoadjuvant therapy can also convert patients from axillary lymph node dissection to sentinel lymph node biopsy [28-30], reducing associated morbidity [26]. From an oncology standpoint, neoadjuvant therapy allows for an in vivo assessment of treatment response while the primary tumor remains intact [25]. Ineffective therapies can be discontinued to avoid the associated toxicities [25], allowing the pre-operative treatment period to be used to investigate novel therapies or treatment combinations [25].

TNBC is very responsive to chemotherapy, with high rates of pathologic complete remission ( $p C R$ ] reported after neoadjuvant treatment [31,32]. In a study of over 1100 patients, Liedtke, et al. [33] found that pCR rates after neoadjuvant treatment were twice as high in TNBC patients as in non-TNBC patients (22\% vs. $11 \%)$. Two separate meta-analyses also found that the absence of ER expression is associated with a greater response to cytotoxic chemotherapy as compared to ER-positive tumors [34,35]. Additionally, the response to neoadjuvant therapy in TNBC tumors can be quite rapid, with Huober, et al. [36] reporting a response after only two cycles of therapy.

The responsiveness of TNBC to neoadjuvant treatment has clinical implications, as studies have demonstrated a clear survival advantage for patients who achieve pCR over those with residual disease after neoadjuvant therapy $[37,38]$. Across all subtypes of breast cancer, the highest rates of $\mathrm{pCR}$ are seen in patients less than 40-years-old, those with higher grade tumors, and those with TNBC; of these factors, hormone receptor status has the strongest predictive value [36]. As younger patients with higher grade tumors are more likely to benefit from neoadjuvant chemotherapy, age and tumor grade can be used to identify TNBC patients who should receive neoadjuvant treatment. Other factors which may encourage the use of neoadjuvant therapy include large tumor size, locally advanced disease, or disease initially ineligible for resection [26].

After completion of neoadjuvant therapy, accurate evaluation of residual tumor size and location is essential for surgical planning [39]. For this purpose, MRI has been shown to be superior to mammography and ultrasound $[39,40]$. While ultrasound and mammography have only modest accuracy for monitoring disease response [41], MRI has been shown to be an excellent modality for assessing both residual disease extent and monitoring early treatment response [39,40,42,43]. A study of 32 patients by Bhattacharrya, et al. [39] demonstrated a strong correlation between preoperative MRI findings and operative histology, with MRI demonstrating a positive predictive value of $99.8 \%$ and a negative predictive value of $80.0 \%$ for the detection of residual invasive disease. 


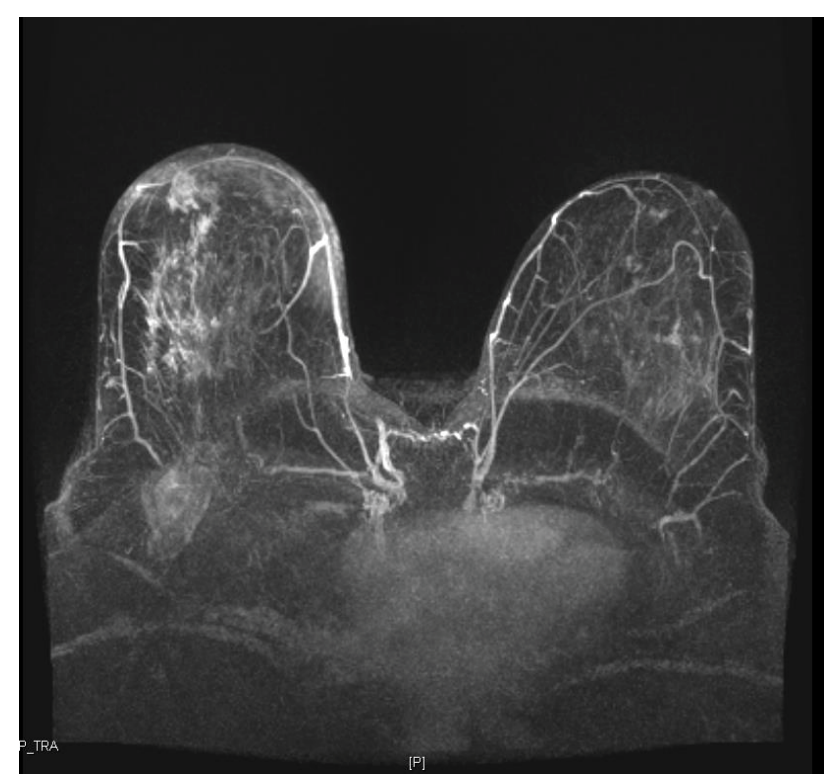

Figure 4: Patchlike residual tumor after neoadjuvant chemotherapy. Post-contrast axial T1 fat-suppressed maximum intensity projection (MIP) images obtained after neoadjuvant chemotherapy of a biopsy-proven triplenegative breast cancer demonstrate a large area of patchy enhancement in the right breast, representing residual tumor. This type of residual tumor is most associated with local recurrence after breast conservation therapy, since there is a greater likelihood of leaving residual tumor with less extensive surgical resections.

The use of $\mathrm{MRI}$ to assess treatment response also necessitates a pre-treatment MRI. TNBC can be classified into several breast cancer phenotypes on pre-treatment MRI which been shown to correlate with response to neoadjuvant therapy $[44,45]$. A study performed by Mukhtar, et al. [44] as part of the I-SPY 1 trial divided tumors into 5 such categories, ranging from a well-defined, unicentric mass to an infiltrative and diffuse tumor (described as "septal spreading"]. The primary endpoint in this trial was clinically meaningful tumor reduction (CMTR], which was defined as a tumor $>4 \mathrm{~cm}$ in size on pre-neoadjuvant therapy MRI which was reduced to $<4 \mathrm{~cm}$ on surgical pathology [44]. Higher rates of CMTR were seen in patients with well-defined tumors, the majority of which were TNBC. In this study, TNBC was also correlated with higher rates of CMTR, independent of tumor phenotype [44]. Well-defined tumors also showed an improved correlation between post-therapy MRI and surgical pathology as compared to the infiltrative, diffuse phenotype [44]; this finding has been supported by other authors [46]. MRI has also been shown to have the greatest accuracy in TNBC as well as HER2 positive tumors [46].

As stated above, one of the goals of neoadjuvant chemotherapy is to reduce the tumor volume and allow for a less extensive surgical resection. Thus, a challenging aspect of surgical planning is determining the amount of breast tissue to be removed and defining the extent of residual tumor. In patients who are or may become candidates for breast conservation therapy, it is imperative to accurately localize the primary tumor; especially in patients expected to have PCR [26]. This is routinely accomplished by placing a radiopaque marker inside the tumor under imaging guidance [47]. Sites of proven tumor pre-treatment are surgically excised to verify treatment response and ensure negative margins, even if no longer evident on post-treatment imaging [48]. Another factor which affects the extent of resection is the pattern of tumor shrinkage [49]. In cases of partial response, three patterns of tumor shrinkage have been described: a solitary residual tumor, patchlike residual tumor (Figure 4), or a main residual tumor with satellite lesions [49]. Of these types, the patchlike lesion is most associated with local recurrence following breast conservation therapy [50], as there is a higher chance of leaving residual tumor cells with less extensive resections [49]. Surgical margins need to be carefully assessed intraoperatively, as the resection of additional tissue may be required for positive margins or patchlike residual tumor [26].

\section{Prognosis}

There is significant variation in the rates of local and distant recurrence among the different breast cancer subtypes, with TNBC reported to have an increased risk of recurrence [51-54] as compared to other breast cancer subtypes. Dent, et al. [55] demonstrated a higher rate of distant recurrence with TNBC, with a recurrence rate of $33.9 \%$ as compared to $20.4 \%$ with other subtypes. A study by Bae, et al. [54] examined 398 women with early stage (stage I or II) TNBC and found several factors associated with local recurrence; these include a family history of breast cancer, lymphovascular invasion, mammographically dense breasts, and the lack of preoperative breast MRI. This study also showed that differences in tumor size and lymph node status, which are important prognostic factors for other breast cancer subtypes, were not associated with significant differences in the recurrence rate of TNBC [54]. The pattern of recurrence is also different for TNBC, with high rates of recurrence seen up to 5 years after diagnosis, followed by a sharp decline in recurrence risk after that time $[54,56]$. In contrast, the risk of recurrence for other breast cancer subtypes is steady and continues for up to 17 years after diagnosis [54-56]. Accordingly, Dent, et al. [55] found a shorter mean time to local recurrence of 2.8 years in TNBC patients as compared to 4.2 years with other subtypes.

The increased recurrence rates with TNBC make proper surgical management of these patients extremely important. As such, understanding the role of pre-operative MRI in these patients is essential. This is especially true in patients who do not undergo neoadjuvant therapy, as these patients would not necessarily have a pre-operative MR study.

The NCl and ACRIN Alliance A011104/ACRIN 6694 trial [57] is attempting to determine the utility of MRI 
in patients at high risk for recurrence, including patients with TNBC (in addition to patients with HER2 amplified tumors). This is a multi-center, randomized controlled trial comparing local recurrence rates after breast conservation therapy in patients who undergo preoperative staging with mammography alone (control) versus mammography and MRI. This study will test the hypothesis that increased rates of multifocality and/or multicentricity account for the higher reported rates of local recurrence in patients with TNBC. If local recurrence rates are higher in the control arm than the $\mathrm{MRI}$ arm, then the occult multifocal/multicentric disease detected by $\mathrm{MRI}$ is clinically relevant. If local recurrence rates are not higher in the control arm, however, then the occult disease detected by MRI is not clinically significant and may be treated by adjuvant radiation and systemic therapy. The results of this trial will have significant implications for the role of pre-operative MRI in TNBC patients.

The long term prognosis of patient's with TNBC is overall poorer than those with non-TNBC [33]. A study of 1,118 breast cancer patients (including 255 with TNBC) at M.D. Anderson Cancer Center demonstrated decreased progression-free and overall survival rates at 3 years for patients with TNBC as compared to those with other breast cancer subtypes [33]; survival rates were similar after 3 years [33]. Multiple factors account for the poorer prognosis in TNBC. TNBC is more likely to have visceral and lung metastases, while non-TNBC more often experience metastatic disease in the bone and skin $[33,58,59]$. TNBC patients are at an increased risk for brain metastases $[31,60,61]$ and TNBC also has a significantly higher nuclear grade [31]. Non-TNBC patients have access to targeted treatments (endocrine therapy, trastuzumab) in addition to chemotherapy, while chemotherapy is essentially the only systemic therapy for TNBC [3]. The prognosis of TNBC is thus largely influenced by response to chemotherapy, with TNBC and non-TNBC patients that achieve pCR having similar survival [33]. Fortunately, TNBC patients are significantly more likely to achieve PCR [33] as compared to those with other breast cancer subtypes.

\section{Conclusions}

TNBC is an aggressive subtype of breast cancer which can demonstrate benign yet particular features on mammography and ultrasound. MRI is the most sensitive imaging test for detection, with TNBC demonstrating more suspicious features on this modality. Chemotherapy is the mainstay of treatment for TNBC, with the majority of patients receiving neoadjuvant treatment. MRI has a proven role in predicting response to chemotherapy based on malignancy phenotype as well as monitoring early treatment response. It also helps with surgical planning after neoadjuvant chemotherapy and the assessment of extent of residual disease. Predicting response to therapy and $\mathrm{PCR}$ is of utmost importance as TNBC patients who achieve pCR after neoadjuvant treatment have improved survival over those with residual disease. Thus, it is important to remember that imaging can supplement the clinical picture, adding vital information pertinent to the diagnosis, treatment and prognosis in this subset of patients.

\section{Conflict of Interest}

The authors have no conflicts of interest to disclose.

\section{References}

1. Spitale A, Mazzola P, Soldini D, Mazzucchelli L, Bordoni A (2009) Breast cancer classification according to immunohistochemical markers: Clinicopathologic features and short-term survival analysis in a population-based study from the South of Switzerland. Ann Oncol 20: 628-635.

2. Dai X, Li T, Bai Z, Yang Y, Liu X, et al. (2015) Breast cancer intrinsic subtype classification, clinical use and future trends. Am J Cancer Res 5: 2929-2943.

3. Dogan BE, Turnbull LW (2012) Imaging of triple-negative breast cancer. Ann Oncol 23.

4. Kim MJ, Ro JY, Ahn SH, Kim HH, Kim SB, et al. (2006) Clinicopathologic significance of the basal-like subtype of breast cancer: A comparison with hormone receptor and Her2/neu-overexpressing phenotypes. Hum Pathol 37: $1217-1226$

5. Fulford LG, Reis-Filho JS, Ryder K, Jones C, Gillett CE, et al. (2007) Basal-like grade III invasive ductal carcinoma of the breast: Patterns of metastasis and long-term survival. Breast Cancer Res 9.

6. Rakha EA, El-Sayed ME, Green AR, Lee AH, Robertson $\mathrm{JF}$, et al. (2007) Prognostic markers in triple-negative breast cancer. Cancer 109: 25-32.

7. Lin NU, Vanderplas A, Hughes ME, Theriault RL, Edge SB, et al. (2009) Clinicopathological features and sites of recurrence according to breast cancer subtype in the National Comprehensive Cancer Network (NCCN). J Clin Oncol 27.

8. Dolle JM, Daling JR, White E, Brinton LA, Doody DR, et al. (2009) Risk factors for triple-negative breast cancer in women under the age of 45 years. Cancer Epidemiol Biomarkers Prev 18: 1157-1166.

9. Millikan RC, Newman B, Tse CK, Moorman PG, Conway $\mathrm{K}$, et al. (2008) Epidemiology of basal-like breast cancer. Breast Cancer Res Treat 109: 123-139.

10. Yang WT, Dryden M, Broglio K, Gilcrease M, Dawood S, et al. (2008) Mammographic features of triple receptornegative primary breast cancers in young premenopausal women. Breast Cancer Res Treat 111: 405-410.

11. Wang $Y$, Ikeda DM, Narasimhan B, Longacre TA, Bleicher RJ, et al. (2008) Estrogen receptor-negative invasive breast cancer: Imaging features of tumors with and without human epidermal growth factor receptor type 2 overexpression. Radiology 246: 367-375.

12. Kojima $Y$, Tsunoda $H$ (2011) Mammography and ultrasound features of triple-negative breast cancer. Breast Cancer 18: 146-151.

13. Dogan BE, Gonzalez-Angulo AM, Gilcrease, Dryden MJ, Yang WT (2010) Multimodality imaging of triple receptornegative tumors with mammography, ultrasound, and MRI. AJR Am J Roentgenol 194: 1160-1166. 
14. Hai-Yan Du, Bao-Rong Lin, Du-Ping Huang (2015) Ultrasonographic findings of triple-negative breast cancer. Int J Clin Exp Med 8: 10040-10043.

15. Lerma E, Barnadas A, Prat J (2009) Triple negative breast carcinomas: Similarities and differences with basal like carcinomas. Appl Immunohistochem Mol Morphol 17: 483494.

16. Boisserie-Lacroix M, Macgrogan G, Debled M, Ferron S, Asad-Syed M, et al. (2013) Triple-negative breast cancers: Associations between imaging and pathological findings for triple-negative tumors compared with hormone receptorpositive/human epidermal growth factor receptor-2negativebreast cancers. Oncologist 18: 802-811.

17. Uematsu T, Kasami M, Yuen S (2009) Triple-negative breast cancer: Correlation between MR findings and pathological findings. Radiology 250: 638-647.

18. Teifke A, Behr O, Schmidt M, Victor A, Vomweg TW, et al. (2006) Dynamic MR imaging of breast lesions: Correlation with microvessel distribution pattern and histologic characteristics of prognosis. Radiology 239: 351-360.

19. Chen JH, Agrawal G, Feig B, Baek HM, Carpenter PM, et al. (2007) Triple-negative breast cancer: MRI features in 29 patients. Ann Oncol 18: 2042-2043.

20. Osman NM, Chalaibi N, Raboh NMA (2014) Triple negative breast cancer: MRI features in comparison to other breast cancer subtypes with correlation to prognostic pathologic factors. Egyptian Journal of Radiology and Nucl Med 45: 1309-1316.

21. Youk JH, Son EJ, Chung J, Kim JA, Kim EK (2012) Triplenegative invasive breast cancer on dynamic contrastenhanced and diffusion-weighted MR imaging: Comparison with other breast cancer subtypes. Eur Radiol 22: 17241734.

22. Leek RD, Landers RJ, Harris AL, Lewis CE (1999) Necrosis correlates with high vascular density and focal macrophage infiltration in invasive carcinoma of the breast. $\mathrm{Br} \mathrm{J}$ Cancer 79: 991-995.

23. Fisher ER, Anderson S, Redmond C, Fisher B (1993) Pathologic findings from the National Surgical Adjuvant Breast Project protocol B-06. 10-year pathologic and clinical prognostic discriminants. Cancer 71: 2507-2514.

24. Stover DG, Bell CF, Tolaney SM (2016) Neoadjuvant and adjuvant chemotherapy considerations for triple-negative breast cancer. Am J Hem Onc 12: 6-12.

25. Thompson M, Moulder-Thompson SL (2015) Neoadjuvant treatment of breast cancer. Ann Surg Oncol 22: 1425-1433.

26. Mamounas EP (2015) Impact of neoadjuvant chemotherapy on locoregional surgical treatment of breast cancer. Ann Surg Oncol 22: 1425-1433.

27. Mieog JSD, Van der Hage Ja, Van de Velde CJ (2007) Neoadjuvant chemotherapy for opérable breast cancer. $\mathrm{Br}$ J Surg 94: 1189-1200.

28. Gianni L, Baselga J, Eiermann W (2002) First report of the European Cooperative Trial in Operable Breast Cancer (ECTO): Effect of primary systemic therapy. Proc Am Soc Clin Oncol 21.

29. Bear HD, Anderson S, Brown A, Smith R, Mamounas $E P$, et al. (2003) The effect on tumor response of adding sequential preoperative docetaxel to preoperative doxorubicin and cyclophosphamide: Preliminary results from National Surgical Adjuvant Breast and Bowel Project Protocol B-27. J Clin Oncol 21: 4165-4174.
30. Wolmark N, Wang J, Mamounas E, Bryant J, Fisher B (2001) Preoperative chemotherapy in patients with operable breast cancer: Nine-year results from National Surgical Adjuvant Breast and Bowel Project B-18. J Natl Cancer Inst Monogr 96-102.

31. Brouckaert O, Wildiers H, Floris G, Neven P (2012) Update on triple-negative breast cancer: Prognosis and management strategies. Int J Womens Health 4: 511-520.

32. Von Minckwitz G, Martin M (2012) Neoadjuvant treatments for triple-negative breast cancer (TNBC). Ann Oncol 23.

33. Liedtke C, Mazouni C, Hess KR, André F, Tordai A, et al. (2008) Response to neoadjuvant therapy and long-term survival in patients with triple-negative breast cancer. $\mathrm{J}$ Clin Oncol 26: 1275-1281.

34. Early Breast Cancer Trialists' Collaborative Group (EBCTCG), Clarke M, Coates AS, Darby SC, Davies C, et al. (2008) Adjuvant chemotherapy in oestrogen-receptor-poor breast cancer: Patient-level meta-analysis of randomised trials. Lancet 371: 29-40.

35. Berry DA, Cirrincione C, Henderson IC, Citron ML, Budman DR, et al. (2006) Estrogen-receptor status and outcomes of modern chemotherapy for patients with node-positive breast cancer. JAMA 295: 1658-1667.

36. Huober J, Von Minckwitz G, Denkert C, Tesch H, Weiss E, et al. (2010) Effect of neoadjuvant anthracycline-taxanebased chemotherapy in different biological breast cancer phenotypes: Overall results from the GeparTrio study. Breast Cancer Res Treat 124: 133-140.

37. Kuerer HM, Newman LA, Smith TL, Ames FC, Hunt KK, et al. (1999) Clinical course of breast cancer patients with complete pathologic primary tumor and axillary lymph node response to doxorubicin-based neoadjuvant chemotherapy. J Clin Oncol 17: 460-469.

38. Guarneri V, Broglio K, Kau SW, Cristofanilli M, Buzdar AU, et al. (2006) Prognostic value of pathological complete response after primary chemotherapy in relation to hormone receptor status and other factors. J Clin Oncol 24: 1037-1044.

39. Bhattacharyya M, Ryan D, Carpenter R, Vinnicombe S, Gallagher CJ (2008) Using MRI to plan breast-conserving surgery following neoadjuvant chemotherapy for early breast cancer. Br J Cancer 98: 289-293.

40. Hylton NM, Blume JD, Bernreuter WK, Pisano ED, Rosen MA, et al. (2012) Locally advanced breast cancer: MR imaging for prediction of response to neoadjuvant chemotherapy--results from ACRIN 6657/I-SPY TRIAL. Radiology 263: 663-672.

41. Chagpar AB, Middleton LP, Sahin AA, Dempsey P, Buzdar $A U$, et al. (2006) Accuracy of physical examination, ultrasonography, and mammography in predicting residual pathologic tumor size in patients treated with neoadjuvant chemotherapy. Ann Surg 243: 257-264.

42. Lobbes M, Prevos R, Smidt M (2012) Response monitoring of breast cancer patients receiving neoadjuvant chemotherapy using breast MRI - a review of current knowledge. J Cancer Ther Res 1: 1-9.

43. McLaughlin R, Hylton N (2011) MRI in breast cancer therapy monitoring. NMR Biomed 24: 712-720.

44. Mukhtar, R, Yau C, Rosen M, Tandon VJ; I-SPY 1 TRIAL and ACRIN 6657 Investigators, et al. (2013) Clinically meaningful tumor reduction rates vary by prechemotherapy MRI phenotype and tumor subtype in the I-SPY 1 TRIAL (CALGB 150007/150012; ACRIN 6657). Ann Surg Oncol 20: 3823-3830. 
45. Esserman L, Kaplan E, Partridge S, Tripathy D, Rugo H, et al. (2001) MRI phenotype is associated with response to doxorubicin and cyclophosphamide neoadjuvant chemotherapy in stage III breast cancer. Ann Surg Oncol 8: 549-559.

46. McGuire KP, Toro-Burguete J, Dang H, Young J, Soran A, et al. (2011) MRI staging after neoadjuvant chemotherapy: does tumor biology affect accuracy? Ann Surg Oncol 18: 3149-3154.

47. Alonse-Bartolome P, Ortega Garcia E, Garijo Avensa F, de Juan Ferre A, Vega Bolivar A (2002) Utility of the tumor bed marker in patient with breast cancer receiving induction chemotherapy. Acta Radiol 43: 29-33.

48. Baron LF, Baron PL, Ackerman SJ, Durden DD, Pope TL Jr (2000) Sonographically guided clip placement facilitates localization of breast cancer after neoadjuvant chemotherapy. AJR Am J Roentgenol 174: 539-540.

49. Wang S, Zhang Y, Yang X, Fan L, Qi X, et al. (2013) Shrink pattern of breast cancer after neoadjuvant chemotherapy and its correlation with clinical pathological factors. World $\mathrm{J}$ Surg Oncol 11: 166.

50. Chen AM, Meric-Bernstam F, Hunt KK, Thames HD, Oswald MJ, et al. (2004) Breast conservation after neoadjuvant chemotherapy: The MD Anderson cancer center experience. J Clin Oncol 22: 2303-2312.

51. Gabos Z, Thoms J, Ghosh S, Hanson J, Deschênes J, et al. (2010) The association between biological subtype and locoregional recurrence in newly diagnosed breast cancer. Breast Cancer Res Treat 124: 187-194.

52. Voduc KD, Cheang MC, Tyldesley S, Gelmon K, Nielsen TO, et al. (2010) Breast cancer subtypes and the risk of local and regional relapse. J Clin Oncol 28: 1684-1691.
53. Nguyen PL, Taghian AG, Katz MS, Niemierko A, Abi Raad RF, et al. (2008) Breast cancer subtype approximated by estrogen receptor, progesterone receptor, and HER-2 is associated with local and distant recurrence after breastconserving therapy. J Clin Oncol 26: 2373-2378.

54. Bae MS, Moon HG, Han W, Noh DY, Ryu HS, et al. (2016) Early stage triple-negative breast cancer: Imaging and clinical-pathologic factors associated with recurrence. Radiology 278: 356-364.

55. Dent R, Trudeau M, Pritchard KI, Hanna WM, Kahn HK, et al. (2007) Triple negative breast cancer: Clinical features and patterns of recurrence. Clin Cancer Res 13: 4429-4434.

56. Foulkes WD, Smith IE, Reis-Filho JS (2010) Triple-negative breast cancer. N Engl J Med 363: 1938-1948.

57. Bedrosian I, Comstock C Effect of preoperative breast mri on surgical outcomes, costs and quality of life in women with breast cancer.

58. Kennecke H, Yerushalmi R, Woods R, Cheang MC, Voduc D, et al. (2010) Metastatic behavior of breast cancer subtypes. J Clin Oncol 28: 3271-3277.

59. Freedman G, Anderson P, Li T, Nicolaou N (2009) Locoregional recurrence of triple-negative breast cancer after breast-conserving surgery and radiation. Cancer 115 : 946-951.

60. Heitz F, Harter P, Traut A, Lueck HJ, Beutel B, et al. (2008) Cerebral metastases $(\mathrm{CM})$ in breast cancer $(\mathrm{BC})$ with focus on triple-negative tumors. J Clin Oncol 26.

61. Dawood S, Broglio K, Esteva FJ, Yang W, Kau SW, et al. (2009) Survival among women with triple receptor-negative breast cancer and brain metastases. Ann Oncol 20: 621627. 